\title{
Propuesta de turismo inclusivo para adultos mayores en la Región de las Altas Montañas
}

\section{Proposal of inclusive tourism for older adults in the High Mountains Region}

BELLATO-GIL, Patricia Lyssett*†, AGUIRRE-MORALES, Fabiola, MENDOZA-LOYO, Octavio Iván y CASTILLO-BLANCO, José Said Universidad Tecnológica del Centro de Veracruz. Ave. Universidad 350 Carretera Federal Cuitláhuac-La Tinaja, Localidad
Dos Caminos, Cuitláhuac, Ver

ID $1^{\text {er }}$ Autor: Patricia Lyssett, Bellato-Gil / ORC ID: 0000-0003-1851-1944, Researcher ID Thomson: X-3120-2018, CVU CONACYT ID: 163879

ID $1^{\text {er }}$ Coautor: Fabiola, Aguirre-Morales / ORC ID: 0000-0002-8212-1505, Researcher ID Thomson: X-2365-2018

ID $2^{\text {do }}$ Coautor: Octavio Iván, Mendoza-Loyo / ORC ID: 0000-0001-6520-0562, Researcher ID Thomson: X-3079-2018

ID $3^{\text {er }}$ Coautor: José Said, Castillo-Blanco / ORC ID: 0000-0002-4846-0693, Researcher ID Thomson: X-2368-2018

DOI: $10.35429 / J T M S .2019 .15 .5 .38 .45$

Recibido 11 de Marzo 2019; Aceptado 30 Junio, 2019

\begin{abstract}
Resumen
Dado el problema mundial de la inversión de la pirámide poblacional, el incremento de adultos mayores puede ser considerado como una alternativa para generar nuevas propuestas de actividades turísticas dirigidas a este segmento, respecto a sus necesidades, intereses y deseos. En México, de acuerdo a INEGI (2015), el índice de envejecimiento es del $38 \%$ con edad promedio de 27 años. Esta alternativa tendrá un impacto social y económico en el sector, haciendo las propuestas necesarias en cuanto a la adaptabilidad de algunos elementos del sistema turístico de los municipios involucrados que conforman la Región de las Altas Montañas. Para ello, en función del objetivo de investigación la metodología que se aplicó es mixta, conformada por una investigación de tipo exploratorio (en donde se recurrió a diversas fuentes documentales y fuentes secundarias), mismas que sirvieron para contextualizar y puntualizar el objeto de estudio, también se llevó a cabo un estudio de tipo descriptivo, mediante la aplicación de 384 encuestas que sirvieron como herramienta preliminar para generar estadísticas y gráficas que concentran los principales indicadores relacionados con el objetivo de estudio. La contribución de este estudio es la inclusión de los adultos mayores con un impacto social y económico en la región.
\end{abstract}

Turismo inclusivo, Adultos mayores, Actividad turística

\begin{abstract}
Given the global investment problem of the population pyramid, the increase in older adults can be considered as an alternative to generate new proposals for tourism activities aimed at this segment, regarding their needs, interests and desires. In Mexico, according to INEGI (2015), the aging rate is $38 \%$ with an average age of 27 years. This alternative will have a social and economic impact on the sector, making the necessary proposals regarding the adaptability of some elements of the tourism system of the municipalities involved that make up the High Mountain Region. To do this, depending on the research objective, the methodology that was applied is mixed, consisting of an exploratory research (where various documentary sources and secondary sources were used), which served to contextualize and point out the object of study, A descriptive study was also carried out, through the application of 384 surveys that served as a preliminary tool to generate statistics and graphs that concentrate the main indicators related to the study objective. The contribution of this study is the inclusion of older adults with a social and economic impact in the region.
\end{abstract}

Inclusive tourism, Older adults, Tourist activity

Citación: BELLATO-GIL, Patricia Lyssett, AGUIRRE-MORALES, Fabiola, MENDOZA-LOYO, Octavio Iván y CASTILLO-BLANCO, José Said. Propuesta de turismo inclusivo para adultos mayores en la Región de las Altas Montañas. Revista Transdisciplinaria de Estudios Migratorios. 2019, 5-15: 38-45

\footnotetext{
*Correspondencia al Autor (correo electrónico: investigacion@utcv.edu.mx)

$\dagger$ Investigador contribuyendo como primer autor.
} 


\section{Introducción}

El turismo en la actualidad es un fenómeno social y económico que en los últimos años ha crecido y beneficiado de manera muy importante en muchos países, incluido México. El turismo en México inició desde la época de la colonia, cuando europeos viajaban a la entonces Nueva España para conocer la gran riqueza de la que hablaban los colonos que volvían a sus países de origen. Esta actividad económica (turismo) se adapta a diferentes necesidades y gustos que el cliente (turista) requiere, es por eso que en todo el mundo podemos encontrar diferentes tipos de turismo.

La evolución del turismo y su relación con la salud física y mental de las personas que lo practican, ha ocasionado que a nivel mundial, se exija un turismo en el que todos los habitantes puedan tener acceso a el mismo; tal es el caso del tema y lema designado por la Organización Mundial de Turismo (OMT) para celebrar el día mundial del turismo en el año 2016: "Turismo Accesible" Turismo para todos; el cual tenía por objetivo promover la accesibilidad universal.

El turismo de la tercera edad, término dado para las personas en edad avanzada, ha tomado mayor impulso en los últimos años, ya que partiendo de que la esperanza de vida del mexicano sobrepasa ya los 75 años (INEGI, 2016), este segmento se posiciona como potencial en la industria turística. La presente investigación tiene como área de estudio la Región de las Altas Montañas en el Estado de Veracruz, conformada por 57 municipios con una riqueza basta en atractivos naturales e históricos, en la cual se posicionan municipios que cuentan con la distinción de "Pueblo Mágico" y empresas turísticas orientadas tanto al turismo de naturaleza como al turismo cultural. Debido al crecimiento notable del turismo en la región mencionada, se proponen actividades orientadas al turismo inclusivo en el segmento de adultos mayores.

El objetivo de esta investigación se fundamenta en la adaptabilidad de la infraestructura existente, así como en las grandes oportunidades para poder albergar a los adultos mayores; al tratarse de un mercado nuevo se requiere analizar las características del entorno y los nuevos perfiles de las personas mayores para determinar las motivaciones que impulsan a viajar a los mismos.

\section{Generalidades}

Región turística de las Altas Montañas

De acuerdo con información obtenida de INEGI, (2010), el estado de Veracruz cuenta con una superficie de $71,826 \mathrm{~km}$. Se localiza en el noreste del país. El clima es cálido subhúmedo y cálido húmedo, principalmente, con una temperatura media anual de 23 grados centígrados, y una precipitación promedio anual de 1,500 mm. Es uno de los estados más representativos de México por su riqueza natural, social, histórica y cultural. Se le considera la puerta de México al mundo, ya que por ahí llegaron los españoles para formar la nación mexicana y hoy es el puerto marítimo comercial más importante del país.

El estado de Veracruz colinda al norte con el estado de Tamaulipas, al sur con los estados de Oaxaca y Chiapas, al poniente con San Luís Potosí, Hidalgo y Puebla y al sureste con el estado de Tabasco. Tiene una superficie de $71,699 \mathrm{~km} 2$, lo que representa el $3.7 \%$ de la superficie del país.

En Veracruz, existe una regionalización turística, definida en la Ley de Turismo del Gobierno Estatal, la cual divide turísticamente al estado en 7 regiones (de norte a sur), encontrando la Región Huasteca, representada principalmente por los municipios de Tuxpan, Chicontepec, Alamo-Temapache, Castillo de Teayo, Pánuco, Tamiahua y Tempoal; la Región Totonaca que abarca principalmente los municipios de Poza Rica, Tecolutla, Cazones y Papantla; la Región Cultura y Aventura, destacando Xalapa, Coatepec, Xico, Perote, Jalcomulco, Actopan, Naolinco; la Región Primeros Pasos de Cortés con los municipios de Veracruz, Boca del Río, La Antigua, Alvarado, Úrsulo Galván, Tlacotalpan. Región de Los Tuxtlas con municipios como: San Andrés Tuxtla, Santiago Tuxtla, Catemaco. Región Olmeca con los municipios de: Minatitlán, Texistepec, Coatzacoalcos y finalmente la Región Altas Montañas que engloba 57 municipios entre los que destacan Orizaba, Fortín de las Flores, Ciudad Mendoza, Atoyac, Zongolica, Yanga, Coscomatepec, Huatusco y Córdoba. 
La Región de las Altas Montañas cuenta con una superficie de 6,053 kilómetros cuadrados, que representan el 8.4 por ciento del territorio estatal, por lo que ocupa el quinto lugar por su extensión territorial, según información de Gobierno del Estado de Veracruz , (2016).

La región presenta un patrón de concentración urbana característico de muchas otras zonas del país y de la entidad. Cuenta con zonas metropolitanas como Córdoba y Orizaba, integradas por 15 municipios que representan menos del $20 \%$ del territorio regional, en los cuales habita $52 \%$ de la población total; por otro lado, en los 42 municipios restantes habita casi la mitad de la población, Gobierno del Estado de Veracruz, (2016)

Además del crecimiento de la población, la Región Turística de las Altas Montañas se caracteriza por el proceso de transición demográfica a partir del envejecimiento de la población. De acuerdo con información de INEGI , (2010), la población infantil se redujo y la población de adultos mayores se incrementó, fenómeno conocido como "inversión de la pirámide poblacional"

Grandes Montañas de Veracruz , (2009) establece que la región de las grandes montañas forma parte de la sierra madre oriental, en ella se localizan el Pico de Orizaba, la montaña más alta de país y el volcán Cofre de Perote. Es la región más montañosa del estado.

Limita al Norte la región del Totonacapan, al sur y al oeste con el estado de Puebla y al este con el Golfo de México y la región de las Llanuras de Sotavento. Su clima es muy variado, predomina el templado húmedo; en las costas es cálido húmedo; en la altiplanicie de Perote es frío y en la cumbre del Pico de Orizaba, muy frío. En esta región nacen ríos importantes: Nautla, Actopan, La Antigua, Jamapa y Blanco.

La vegetación es muy variada, en las partes bajas hay encinos; más arriba cedros y liquidámbares; en las faldas de las montañas, pinos, abetos y oyameles. En las partes más elevadas predomina el Zacatón. Entre su fauna representativa destacan ardillas, zorras, mapaches, venados, tejones, tlacuaches, puercoespines, cacomixtles, armadillos, culebras, palomas, pericos, codornices, zopilotes, chachalacas y búhos.
El relieve de las grandes montañas permite una gran variedad en cultivos. En las partes baja se siembra maíz, fríjol, caña de azúcar, naranja, mango, y plátano. En terrenos altos, trigo, papa, haba, alfalfa, manzana, durazno, aguacate y nogal. Sobresale la producción de café en Coatepec, Córdoba, Huatusco, Teocelo y otros municipios.

En esta región existe principalmente la crianza de vacas, ovejas, cabras, cerdos, caballos $\mathrm{y}$ mulas. Se han desarrollado importantes industrias: beneficios de café, pasteurizadoras de leche, fábricas de cerveza, hilados y tejidos, cemento, refrescos, papel y jabón. También existen ingenios azucareros, como El Potrero, Mahuixtlán, La Concepción y El Miguelito.

En esta región se ubican dos grandes y antiguas Áreas Naturales Protegidas de interés de la federación: parte del Parque Nacional Pico de Orizaba (con 18,750 ha de superficie) y el Parque Nacional Cañón del Río Blanco (con 55,690 ha de superficie). Recientemente, en 2013, el Gobierno del estado de Veracruz, decretó un Área Natural Protegida de nombre Metlac-Río Blanco, con una superficie de 31,790 ha, la cual se sobrepone, en parte, al polígono del Parque Nacional Cañón del Río Blanco, Rivera, (2015)

En términos históricos, esta región también es importante, ya que desde tiempos de la conquista ha funcionado como zona de paso entre la Ciudad de México (antes la Gran Tenochtitlán) y el Puerto de Veracruz (antes de la Vera Cruz), además de que existen una gran cantidad de fincas o haciendas cafetaleras de la época de la colonia en toda la región. Naveda, (1997)

La región de las Altas Montañas es una zona con un gran valor biocultural y paisajístico que ofrece un alto potencial para una muy diversa oferta ecoturística de primer nivel. Desde el punto de vista natural o biológico, la región resguarda una muy rica biodiversidad, demostrada por las casi 400 especies de aves, las casi 2000 especies de plantas nativas y naturalizadas (Rivera, 2015) 
En la Región Turística de las Altas Montañas existen varios municipios con características idóneas para el desarrollo del turismo cultural y de naturaleza, ya que se cuenta con una oferta de atractivos diversificada, lo que genera la demanda de actividades turísticas principalmente en época vacacional y fines de semana. En cada municipio existen todavía algunas localidades de los municipios de Zongolica, Yanga, Ciudad Mendoza, Ixtaczoquitlán, Coetzala y Amatlán de los Reyes, que aún resguardan la cultura náhuatl característica de esta región, existen vestigios arqueológicos de importancia y con potencial turístico para nacionales y extranjeros.

Por otra parte, la oferta cultural se ve incrementada con cada una de las festividades de los municipios de la región como por ejemplo las fiestas de la cruz y del rosario en Amatlán de Los Reyes, el carnaval afromexicano en Yanga, las fiestas de la Covadonga en Córdoba, la fiesta de San José en Atoyac, por mencionar algunas y de manera general la celebración de los fieles difuntos, las fiestas patronales de las localidades, la música tradicional y una gastronomía típica de cada zona. En diversos municipios de la sierra de Zongolica viven indígenas nahuas. Generalmente en esta región abundan las fiestas y tradiciones, destacan las ferias de Coatepec, Fortín, Xalapa y Xico. Son famosas las danzas de los Santiagos en Naolinco y la Malinche en Acatlán.

\section{Objetivo General}

Identificar la viabilidad del turismo inclusivo a partir del estudio de las preferencias de viaje de adultos mayores de la Región de las Altas Montañas para la propuesta de actividades turísticas enfocadas a este segmento.

\section{Fundamentación Teórica}

En el estudio teórico del presente trabajo, se encontraron investigaciones y estudios principalmente en España y México respecto a la adaptación e inclusión de los adultos mayores en actividades turísticas. Según la Organización Mundial del Turismo (OMT), el turismo se debe entender como el conjunto de actividades que realizan las personas durante sus viajes y estancias en lugares distintos al de su entorno habitual, por un periodo inferior a un año, por motivos no relacionados con el ejercicio de una actividad remunerada en el lugar visitado.
La industria internacional del turismo es el mayor proveedor de empleos del planeta $\mathrm{y}$ cuenta con variedad de actores heterogéneos (Buhalis \& Law, 2008). Es una de las industrias más dinámicas por su importancia en la movilización de personas por todo el mundo y su contribución en términos económicos, ambientales y socioculturales (Ministerio de Comercio Industria y Turismo, 2011); genera oportunidades de negocio, ingresos por divisas, contribuye al ingreso público y privado, estimula la creación de tecnologías y la formación de capital humano (Monterubbianesi \& Zapata, 2011)

"Turismo es el conjunto de relaciones y fenómenos que se producen como consecuencia del desplazamiento y estancia temporal de personas fuera de su lugar de residencia, siempre que no esté motivado por razones lucrativas" (Hunziker \& Krapf, 1942), en otras palabras, el turismo es una práctica social colectiva que integra mecanismos distintos de relación con el espacio, la identidad y el ocio, por eso, es una práctica generadora de actividad económica.

El termino de turismo para todos se hace presente por primera vez en la campaña Tourism for all, llevada a cabo en Reino Unido en 1989, el cual define a esta actividad como: "Aquella forma de turismo que planea, diseña y desarrolla actividades turísticas de ocio y tiempo libre de manera que puedan ser disfrutadas por toda clase de personas con independencia de sus condiciones físicas, sociales o culturales" World Tourism Organization UNWTO, (2014).

El turismo social ha evolucionado a paso lento y ahora se busca el bienestar de no solo las personas de la tercera edad y los obreros; sino también de las personas que cuentan con algún tipo de discapacidad. Existen algunos países europeos como España, que trabaja a favor de la población y se encamina para ser un país que se ocupa en esta problemática CECIES Pensamiento Latioamericano y Alternativo, (2019)

Un ejemplo de ello es el Plan Nacional de Accesibilidad 2004 -2012 de Cádiz, España en donde se enfatiza en la accesibilidad para toda la sociedad de este país; además de la construcción y creación de espacios como jardines, calles y edificios para que todas las personas puedan desplazarse libremente (Universidad de Cádiz, 2009) 
El fenómeno del envejecimiento de la población ha sido analizado desde las particularidades de las tendencias de fecundidad, mortalidad y migración. En muchos estudios se observan particularmente los cambios en la transición demográfica y se hace énfasis en la necesidad de discutir sobre los escenarios demográficos futuros en relación con los proyectos de desarrollo económico y social de México y América Latina (Ordorica Mellado, 2004)

La mayor parte de la población de adultos mayores tiene más tiempo de ocio ya que de los 65 años en adelante en su mayoría son jubilados o no tienen un trabajo fijo. El ocio puede definirse como el tiempo libre o descanso que las personas puedan tener en determinado tiempo.

La construcción de una sociedad inclusiva y del turismo accesible, depende de que el público permita opciones turísticas donde se asegure que todas las personas, en este caso los adultos mayores tengan igualdad de oportunidades para ejercer estas actividades tanto en el espacio urbano como en el espacio rural, además se deberían establecer normas de convivencia y receptividad para la sociedad, ya que, en su mayoría, no comprenden la importancia de estas personas. (Brinckmann \& Jairo, 2003).

La mayoría de las investigaciones afirman que los adultos mayores expresan preocupación por la serie de cambios que se han producido (Cabrera Acevedo, 1998), tanto sociales como políticos, económicos y culturales que a su vez han producido variaciones en las formas de vida de los mayores, su visión de la misma, sus comportamientos, actitudes, hábitos, intereses, gustos e incluso de la propia identidad, respecto a las generaciones anteriores. (Alén González, Dominguez Vila, \& Fraíz García , 2010) Recientemente en Madrid (2002), se realizó un foro sobre "Viajes y tercera edad" donde se presentaron las experiencias de diferentes países y se abordaron los beneficios de dichos programas, como sacar a los adultos mayores del aislamiento, luego de jubilarse; devolverles el placer de desplazarse para preservar el mayor tiempo posible tanto su independencia física como social; integrarlos en una actividad grupal a fin de que renueven el contacto con los demás.
La mayoría de las personas expresó su necesidad de continuar trabajando una vez jubiilado, para poder compensar la reducción de sus ingresos y así continuar con el estilo de vida que llevaba (Marín L. , y otros, 2004)

En México en las décadas recientes, la sociedad de consumo y la globalización de los mercados, la búsqueda de nuevos empleos y la búsqueda de la calidad han generado importantes competencias entre las empresas turísticas (Hill \& Jones, 1999) posibilitando nuevas opciones de ocio y tiempo libre, lo cual ha logrado el despegue del turismo. Esta actividad se ha convertido en el mayor sector económico a distintas escalas: nacionales, regionales y locales. Un ejemplo en el año 2018, el turismo contribuyó con 17.2\% del PIB de México (Vanguardia, 2019). La actividad turística, se adecua constantemente a los cambios sociales y a las demandas del mercado.

En esta nueva oferta turística se está imponiendo una forma de hacer turismo, con el respeto a la protección del medio ambiente (Casanueva Rocha, García del Junco, \& Caro González , 2000) y la sustentabilidad, algunas de ellas han sido, el turismo rural y el ecoturismo (Boo, 1990). Y así sucesivamente seguirán surgiendo diferentes destinos y actividades turísticas pero el propósito ahora es que los adultos mayores tengan una mayor participación en él, donde se sienten excluidos y apoyándolos así, a tener una mejor calidad de vida. En este sentido, el turismo rural también puede ser un complemento importante para las débiles economías locales y un detonante para el desarrollo regional que impulse otros sectores, como el primario, al tiempo que mejore las condiciones de vida de sus habitantes y los adultos mayores. También es importante que las políticas sociales vayan encaminadas a favorecer el autoempleo. Algunos investigadores (Dachari, Orozco, \& Arnaiz, 2005)indican que, si el turismo para adultos mayores, como podría ser el rural, no se planifica, se corre el riesgo de pasar de un turismo de baja intensidad a uno masivo e insostenible, algunas opciones para evitarlo son: el promover distintos productos turísticos adaptados a las diferentes estaciones del año, además, difundir los espacios naturales, creando rutas turísticas por los municipios, rehabilitar centros turísticos, senderos de flora y fauna, casas y albergues rurales. 
Así como actividades al aire libre realizando mayores esfuerzos para atraer inversores y turistas de los centros urbanos más importantes del estado e impulsar el turismo proveniente del país vecino, ya que es el principal emisor de turistas a México, con $88.2 \%$ sobre todo estadounidenses que tiene antepasados y familiares mexicanos (SECTUR, 2004). Al igual que mejorar las vías de comunicación, tanto caminos rurales, como carreteras secundarias, estatales y federales. Para lograrlo sin ningún daño, sería necesaria una organización previa de las actividades turísticas a realizar en cada municipio, contar con la inversión que debe provenir del capital público, puede ir acompañada de ayudas económicas y fiscales a la inversión privada ya que sería obligatorio el establecimiento de una infraestructura e infraestructura turística mínima.

\section{Metodología}

El presente estudio es de tipo transversal ya que se hizo un corte único en el tiempo. La metodología es de tipo exploratoria, descriptiva y documental ya que se realizó la recopilación de la literatura a partir de fuentes secundarias escritas. Se diseñó y aplicó un instrumento de investigación cuantitativo dirigido a una muestra de 329 elementos de estudio, en los cuales se consideró la variable edad, ya que fue dirigido a adultos de clase media a media alta y de edades entre 50 y 90 años de diversos municipios de la región turística de las Altas Montañas ya que, de acuerdo al objetivo planteado para esta investigación, se busca conocer si los adultos mayores que oscilan entre esas edades les gustaría viajar y si es así, qué tipo de turismo es el que prefieren para que con dichos resultados se lleven a cabo propuestas de actividades de turismo inclusivo para adultos mayores. También se utilizó el coeficiente alfa de Cronbach para conocer el grado de confiabilidad $^{1}$ de la aplicación del instrumento. Se obtuvieron datos que fueron procesados a partir del software SSPS, mismo que fue utilizado para realizar la captura y análisis de los datos obtenidos.

\footnotetext{
${ }^{1}$ La confiabilidad o fiabilidad, se refiere a la consistencia o estabilidad de una medida. Una definición técnica de confiabilidad que ayuda a resolver tanto problemas teóricos como prácticos es aquella que parte de la investigación de qué tanto error de medición existe en un instrumento de medición, considerando tanto la varianza sistemática como la varianza por el azar, Kerlinger, (2002)

El SPSS $^{2}$ es conocido por su capacidad de gestionar grandes volúmenes de datos y es capaz de llevar a cabo análisis de texto entre otros formatos más. Finalmente se emitieron conclusiones y recomendaciones acerca de las posibilidades del turismo inclusivo en la región de las Altas Montañas.

\section{Resultados}

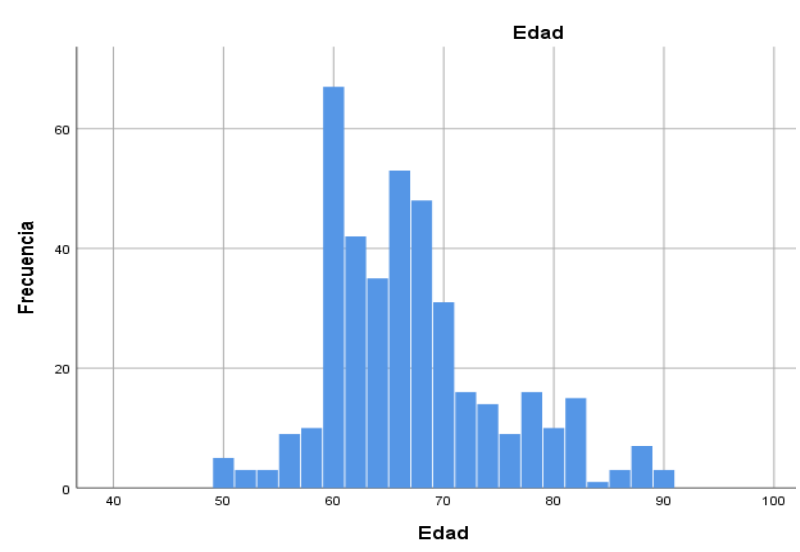

Gráfica 1 Edad

Fuente: Elaboración Propia Bellato, P. (2019)

En la gráfica 1 se puede observar que el rango de edad más frecuente de los individuos que formaron parte de la muestra oscila entre 60 y 70 años de edad.

\begin{tabular}{|l|l|r|r|r|r|}
\hline \multicolumn{7}{|c|}{ ¿Le gusta viajar? } \\
\hline \multirow{3}{*}{ Válido } & Sí & Frec. & \multicolumn{1}{c|}{$\%$} & \% válido & \% acum \\
\cline { 2 - 6 } & No & 346 & 86.5 & 86.7 & 86.7 \\
\cline { 2 - 7 } & Total & 399 & 13.3 & 13.3 & 100.0 \\
\hline Perd. & Sistema & 1 & .3 & & \\
\hline Total & 400 & 100 & & \\
\hline
\end{tabular}

Tabla 1 Gusto por viajar

Fuente. Bellato, P. (2019)

En la tabla 1 se observa que más de la mitad de los encuestados gustan de viajar $(86.5 \%)$, en contraste con el $(13.3 \%)$ que asegura una respuesta negativa.
2 SPSS es un software utilizado para realizar la captura y análisis de datos para crear tablas y gráficas con data compleja. QuestionPro, (2019) 


\begin{tabular}{|c|c|c|c|c|}
\hline \multicolumn{5}{|c|}{ Cuando usted viaja, ¿de qué forma lo hace? } \\
\hline & Frec. & $\%$ & \% válido & $\%$ acum \\
\hline & Solo & 64 & 16.0 & 17.7 \\
\hline & Con un familiar & 116 & 29.0 & 32.0 \\
\hline & $\begin{array}{l}\text { Con dos o más } \\
\text { familares }\end{array}$ & 153 & 38.3 & 42.3 \\
\hline & Excursiones & 20 & 5.0 & 5.5 \\
\hline & Con amigos & 9 & 2.3 & 2.5 \\
\hline & Total & 362 & 90.5 & 100.0 \\
\hline Perd. & Sistema & 38 & 9.5 & \\
\hline & Total & 400 & 100 & \\
\hline
\end{tabular}

Tabla 2 Acompañamiento de viaje

Fuente. Bellato, P. (2019)

En la tabla 2 se observa que el mayor acompañamiento de viaje es a partir de dos o más familiares con un $38 \%$

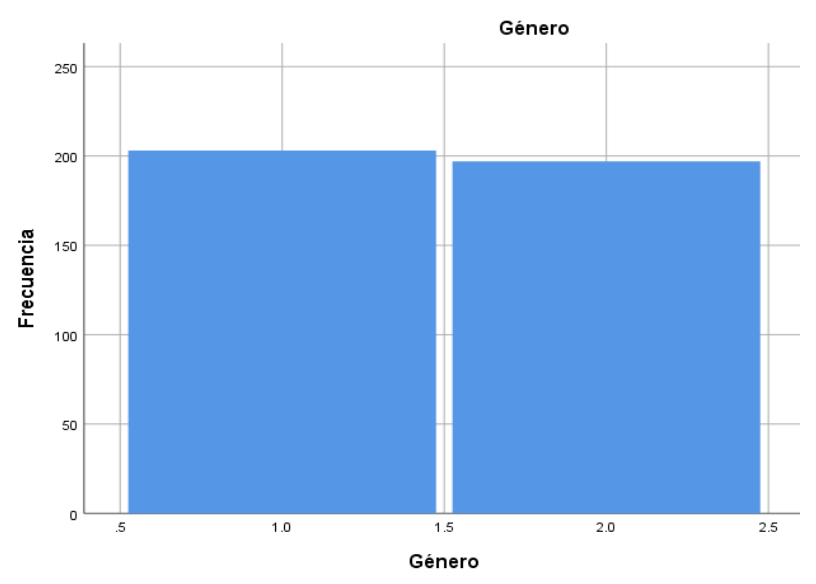

Gráfica 2 Género

Fuente. Elaboración propia Bellato, P. (2019)

\begin{tabular}{|l|l|r|r|r|r|}
\hline \multicolumn{7}{|c|}{ Género } \\
\hline \multirow{3}{|c|}{} & Frec. & \multicolumn{1}{|c|}{$\%$} & $\%$ válido & $\%$ acum. \\
\hline Válido & Fem. & 203 & 50.7 & 50.7 & 50.7 \\
\cline { 2 - 6 } & Masc. & 197 & 49.3 & 49.3 & 100.0 \\
\cline { 2 - 6 } & Total & 400 & 100.0 & 100.0 & \\
\hline
\end{tabular}

Tabla 3 Género

Fuente. Bellato, P. (2019)

\section{Análisis de resultados}

Con los resultados obtenidos en la variable edad, el mayor número de encuestados oscila entre los 60 y 70 años, siendo el género femenino el predominante; en casi su totalidad les gusta viajar y la mayoría de ellos lo hace acompañado por dos o más familiares. El gusto por las actividades turisticas está orientado a: turismo religioso, ecoturismo, turismo rural, turismo cultural, turismo de salud, turismo de sol y playa, turismo holístico, turismo de negocios y turismo educacional.
El presupuesto que destinan para gastar este segmento oscila entre los $\$ 5,000.00$ y $\$ 10,000.00 \mathrm{MXN}$. Suelen viajar ocasional y frecuentemente utilizando servicios turisticos de hospedaje y restauración. Prefieren visitar centros históricos de las ciudades y comunidades cercanas a las mismas. En su mayoria eligen los viajes nacionales y los realizan de 2 a 5 veces al año.

\section{Conclusión}

Con la realización de esta investigación se concluye que el turismo accesible es una tendencia actual en la región de las Altas Montañas, el cual específicamente en el segmento de adultos mayores encuentra oportunidades de desarrollo. Las actividades orientadas a la naturaleza y la cultura son las que generan mayor interés, por lo que la diversificación de la oferta para este segmento tiene que ser adaptada a los gustos y preferencias, así como tomar en cuenta las motivaciones de viaje de las personas en plenitud. La consistencia interna del instrumento de medición al indicarse como buena, otorga la confiabilidad para proponer productos turísticos especializados en el ocio y recreación.

En los municipios con viabilidad para el desarrollo de turismo de naturaleza se determina que las actividades implícitas en el turismo rural son las preferidas para el nicho de estudio. Los municipios con riqueza cultural son sin duda también los escogidos por estas edades, ya que son muchos los atractivos en esta región que cuentan con declaratorias históricas por el Instituto Nacional de Antropología e Historia (INAH). Es necesario realizar las adaptaciones pertinentes de espacios, señalética y fuentes de información tanto para la población receptora como para los prestadores de servicios turísticos; así como promover programas y políticas públicas en la región, que permitan la planeación y desarrollo del turismo accesible para adultos mayores en la delimitación territorial en la que fue realizada la investigación.

\section{Referencias}

Alén González, M., Dominguez Vila, T., \& Fraíz García , A. (2010). El turismo senior como segmento de mercado emergente. Cuardenos de turismo, 9-24. 
Boo, E. (1990). Ecoturismo: Potenciales y Escollos.

Brinckmann, W., \& Jairo, S. (2003). Desafíos para los estudiosos del turismo: la construcción de la «sociedad inclusiva» y del «turismo accesible». Cuadernos de turismo, 41-58.

Buhalis, D., \& Law, R. (2008). Progress in information technology and tourism management: 20 years on and 10 years after the Internet-The state of eTourism research. Tourism Management, 609-623.

Cabrera Acevedo, G. (1998). La siguiente etapa de las políticas de población. Papeles de población, 39-44.

Casanueva Rocha, C., García del Junco, J., \& Caro González , F. (2000). Organización y gestión de empresas turísticas. España: Pirámide

CECIES Pensamiento Latioamericano y Alternativo. (2010-2019). CECIES Pensamiento Latioamericano y Alternativo. Retrieved from CECIES Pensamiento Latioamericano y Alternativo:

http://www.cecies.org/articulo.asp?id=372

Dachari, A., Orozco, J., \& Arnaiz, S. (2005). Retos del Turismo Rural en América Latina. Desarrollo Rural y Turismo.

Gobierno del Estado de Veracruz . (2016). Programas regionales veracruzanos. Retrieved from file://C:/Users/Octavio/Desktop/tf07-prmontana.pdf

Grandes Montañas de Veracruz . (2009). Las Grandes Montañas . Retrieved from http://benvregiover.blogspot.com/2009/06/las-grandesmontanas.html

Hill, C., \& Jones, G. (1999). Administración Estratégica . Bogotá: McGraw-Hill.

Hunziker, W., \& Krapf, K. (1942). Serie de publicaciones del seminario para el estudio en la Handels-Hochschule St. Gallen. Zurich.

INEGI - (2010). Retrieved from file://C:/Users/Octavio/Desktop/tf07-prmontana.pdf
INEGI . (2010). Secretaría de Economía. Retrieved from Información económica y estatal Veracruz

https://www.gob.mx/cms/uploads/attachment/fi le/97626/veracruz.pdf

Kerlinger, F. (2002). Investigación del comportamiento. In Métodos de investigación en ciencias sociales. McGraw Hill. Retrieved from Métodos de investigación en ciencias sociales.

Marín L. , P., Carrasco G. , M., Cabezas U, M., Gac E., H., Hoyl M., T., Duery D, P., . . . Dussaillant K, C. (2004). Impacto biomédico de los viajes en los adultos mayores chilenos. Rev. Med. Chile, 573-578.

Monterubbianesi, J., \& Zapata, S. (2011). Impactos del turismo sobre el crecimiento económico y el desarrollo. El caso de los principales destinos. Pasos, 291-303.

Naveda, A. (1997). La villa de Córdoba a finales de la colonia. Sotavento 2.

Ordorica Mellado, M. (2004). Cambios demográficos y desafíos para la política de la población en México. Una reflexión a largo plazo. Papeles de población, 14-24.

QuestionPro. (2019). ¿Qué es SPSS y cómo utilizarlo? . Retrieved from https://www.questionpro.com/es/que-esspss.html

Rivera, J. (2015). Flora, vegetación y priorización de áreas de conservación del Parque Nacional Cañón del Río Blanco. In Tesis doctoral en ciencias naturales para el desarrollo . Costa Rica .

Vanguardia. (2019, Marzo 1). Vanguardia. Retrieved from Vanguardia: https://vanguardia.com.mx/articulo/sector-deviajes-y-turismo-crecio-mas-que-el-pib

World Tourism Organization UNWTO. (2014). World Tourism Organization UNWTO. Retrieved from World Tourism Organization UNWTO: https://www.eunwto.org/doi/pdf/10.18111/9789284416226 